\title{
The 750kV Transformer Condition Assessment Based on Improved Grey-Target
}

\author{
$\mathrm{Xi} \mathrm{He}^{1, *}$, Ruiping Zhang ${ }^{1}$ and Haiying Dong ${ }^{2}$ \\ ${ }^{1}$ School of Automation and Electrical Engineering, Lanzhou JiaoTong University, Lanzhou 730070, P.R.China \\ ${ }^{2}$ School of New Energy and Power Engineering, Lanzhou JiaoTong University, Lanzhou 730070, P.R.China \\ ${ }^{*}$ Corresponding author
}

\begin{abstract}
In view of the $750 \mathrm{kV}$ transformer that has high voltage, high insulation level, and heavy load all the years.This paper analysis the monitor data to obtain characteristic parameters which can reflect the transformer healthy conditions. On the basis of traditional Grey target theory, It uses the improved Grey target theory to give the different weight of each index, and calculates the approaching degree to make health assessment, so as to obtain the purpose of determine transformer-health.
\end{abstract}

Keywords-grey target theory; condition assessment; $750 \mathrm{kV}$ transformer

\section{INTRODUCTION}

The breakdown of transformer can reduce the reliability about electrical power system, and cause great economic loss. $750 \mathrm{kV}$ transformer is one of the core equipment in the western power transmission. Its running status concerns regional substation or even the whole northwest grid power system security, stability and economic operation. How to evaluate the aging degree of transformer insulation need a certain technical basis. For this, some scholars introduce the Grey target theory to condition assessment of power transformer. This theory take the uncertainty system as the object to study. It can extract valuable information, which based on the "part" of the known information of generation and development, to achieve a correct description and effective monitoring about system behavior and evolution. When the breakdown occurs, the $750 \mathrm{kV}$ transformers are often accompanied by multiple state changes, which is a typical 'Grey system'. Compared with the common transformer, its experimental project and the monitor quantity are more. At present, home and abroad, where have no authoritative reference rules. Thus, we needs further study in this field, and new methods and ideas are should be put forward[1-6].

\section{THE SELECTION OF TRANSFORMER CONDITION ASSESSMENT INDICATORS}

Making the condition assessment to insulation system of $750 \mathrm{kV}$ transformer, we mainly use the factors which affect its insulation state for diagnosis and analysis. These factors such as the analysis data of high performance liquid chromatography, the analysis data of oil chromatogram and the data of electrical test project, the oil project analysis, operating record, overhaul history, appendix condition, appearance inspection, movement environment and so on[7-8].
Which can from the different side, the varying degree and the different level reflect the transformer healthy condition.

\section{A. High Performance Liquid Chromatography (HPLC) Analysis}

The solid insulation material of oil-immersed transformer is insulating paper mainly, and the main ingredients of insulating paper is cellulose. Due to the temperature, moisture, oxygen and the oil of acidic compounds combined effect, the cellulose can degrade to produce a series of oxygen heterocyclic compounds, which will dissolve in insulating oil when transformer operates. Furfural is the major product[9]. We can analysis insulating paper fiber aging by furfural content.

\section{B. Oil Chromatograph Analysis Data}

Large oil-immersed $750 \mathrm{kV}$ transformer uses its oil as dielectric and heat dissipation. During normal operation, dissolved gases in transformer's oil is mainly oxygen and nitrogen. When transformer breaks down or the unusual situation. The oil will decompose some characteristic gas, which Generally have $\mathrm{H}_{2}, \mathrm{CO}, \mathrm{CO}_{2}, \mathrm{CH}_{4}, \mathrm{C}_{2} \mathrm{H}_{6}, \mathrm{C}_{2} \mathrm{H}_{4}, \mathrm{C}_{2} \mathrm{H}_{2}$ [10].We can use the abnormal gas data as $750 \mathrm{kV}$ transformer breakdown characteristics, by analysis the oil-gas component and content to assess its running state.

\section{The Electrical Test Analysis Data}

Under the action of external high voltage or strong electric field, the transformer will be aging. For transformer in operation, If the results of chromatographic analysis show the abnormal state of the transformer, it indicates that there may be a discharge of the transformer. We can make an in-depth analysis by partial discharge test.

\section{HeAlth CONDition Assessment OF TRANSFORMER WITH TRADITIONAL GREY TARGET THEORY}

The basic idea of grey target theory is to set a grey target in the condition which has no standard mode, and to find the bull 's-eye in grey target. it will get the approaching degree by evaluation index and target sequence comparison. Finally, determine the assessment level with target classification principles[11]. Because it can identify the transformer's insulation state without the standard model, it is feasible to evaluate the health status of the transformer. 


\section{A. Selection of Standard Model}

In this paper, we take the transformer normal state index as the grey target approaching.

We set $v_{i}$ as the transformer $i$ state model, $v(k)$ as the $k$ index sequence, select $v_{0}(k)$ for the standard model. The formula is as follows:

$$
v_{0}(k)= \begin{cases}\max _{i} v_{i}(k), & P O L(v(k))=P O L(\max ) \\ \min _{i} v_{i}(k), & P O L(v(k))=P O L(\min )\end{cases}
$$

$\operatorname{POL}(v(k))=P O L(\max ), P O L(v(k))=P O L(\min )$ which have the properties of the maximum and minimum values of the $k$ state.

\section{B. Unified Measure Transformation}

The each state of transformer needs to be unified into the same measure. Otherwise, small numerical functions will be covered by large numerical. When the huge difference between the two data. Uniform measure transformation is as follows:

$$
E\left(v_{i}(k)\right)=\frac{\min \left\{v_{i}(k), v_{0}(k)\right\}}{\max \left\{v_{i}(k), v_{0}(k)\right\}}=\omega_{i}(k)
$$

In the formula, $\omega_{i}(k) \in[0,1]$ as transform values.

\section{The Calculation of Transformer Approaching Degree}

Approaching degree obtained is the core problem of grey target theory, Both the closer, the higher the degree of target.

The difference information space is:

$$
\Delta_{0 i}(k)=\left|\omega_{i}(k)-\omega_{0}(k)\right|=\left|1-\omega_{i}(k)\right|
$$

$\left.\gamma\left(\omega_{0}(k), \omega_{i}(k)\right)\right)^{---t h e ~ t a r g e t ~ c o e f f i c i e n t ~ w h i c h ~ c a n ~ b e ~ e x p r e s s e d ~}$ as:

$$
R\left(\omega_{0}(k), \omega_{i}(k)\right)=\frac{\min _{i} \min _{k} \Delta_{0 i}(k)+\rho \max _{i} \max _{k} \Delta_{0 i}(k)}{\Delta_{0 i}(k)+0.5 \max _{i} \max _{k} \Delta_{0 i}(k)}
$$

Approaching degree for:

$$
R\left(\omega_{0}, \omega_{i}\right)=\frac{1}{n} \sum_{k=1}^{n} R\left(\omega_{0}(k), \omega_{i}(k)\right)
$$

In the formula, $\rho$ as judgment for decision maker.

\section{Determination of Health Status of Transformer}

The level of evaluation objects is decided by the approaching degree. When $\min \min _{k} \Delta_{0 i}(k), \rho=0.5$, there are:

$$
0.333333=\frac{\rho}{1+\rho} \leq R\left(\omega_{0}(k), \omega_{i}(k)\right) \leq 1
$$

Corresponding the Table I[12], we will make the target ladder on the basis of the practical significance, and according the classification strategy to determine the health status of the transformer.

TABLE I. APPROACHING DEGREE GRADING AND MAINTENANCE RECOMMENDATIONS

\begin{tabular}{|c|c|c|c|c|}
\hline $\begin{array}{c}\text { approaching } \\
\text { degree level }\end{array}$ & {$[0.33,0.4)$} & {$[0.4,0.53)$} & {$[0.53,0.65)$} & {$[0.65,1]$} \\
\hline $\begin{array}{c}\text { Transformer's } \\
\text { condition }\end{array}$ & serious & abnormal & $\begin{array}{c}\text { Pay attention } \\
\text { to }\end{array}$ & normal \\
\hline $\begin{array}{c}\text { Maintenance } \\
\text { advice }\end{array}$ & $\begin{array}{c}\text { as soon as } \\
\text { possible }\end{array}$ & Timely & $\begin{array}{c}\text { Not more } \\
\text { than the } \\
\text { normal cycle }\end{array}$ & $\begin{array}{c}\text { normal } \\
\text { cycle }\end{array}$ \\
\hline
\end{tabular}

\section{HEALTH CONDITION ASSESSMENT OF TRANSFORMER} WITH IMPROVED GREY TARGET THEORY

The traditional grey target theory shows that the approaching degree is the average of the corresponding coefficient of the different status of the target. It regards the influence of different indexes of approaching degree as the same. But in fact, each index is different to the approaching degree, especially $750 \mathrm{kV}$ transformer, which experimental project and the monitor condition is more. When fault occurs, it is often accompanied by multiple state variables, each state variable will make different influence for evaluation results. To ensure the quality of evaluation, we can according to the influence degree of the evaluation result to assign different weights for various performance index, so as to achieve the objective and fair evaluation results.

\section{A. The Use of Analytic Hierarchy Process (AHP) to Determine the Static Weights}

Transformer condition evaluation index contains both quantitative information and qualitative information. When analytic hierarchy process is used to analysis the date, it can combining qualitative and quantitative, so it is suitable for $750 \mathrm{kV}$ transformer condition assessment[13].

\section{1) Constructing expert judgment matrix}

We use analytic hierarchy process to make pairwise comparison about quantity of state. and according to them relative importance to construct judgment matrix.

Suppose the evaluation model contains $m$ states. According to the result of comparing two important degree, we can get the judgment matrix. 


$$
D=\left[\begin{array}{ccccc}
\frac{d_{1}}{d_{1}} & \frac{d_{1}}{d_{2}} & \ldots & \ldots & \frac{d_{1}}{d_{m}} \\
\frac{d_{2}}{d_{1}} & \frac{d_{2}}{d_{2}} & \ldots & \ldots & \frac{d_{2}}{d_{m}} \\
\dddot{d_{m}} & \ldots & \ldots & \ldots & \ldots \\
\frac{d_{m}}{d_{1}} & \frac{\ldots}{d_{2}} & \ldots & \ldots & \frac{d_{m}}{d_{m}}
\end{array}\right]
$$

In the formula, $d_{i} / d_{j}\left(1<d_{i} / d_{j}<9\right)$ is the number which woke out by specialist whom according to the importance degree of the first $\mathrm{i}$ condition quantity to the first $\mathrm{j}$ condition quantity.

\section{2) Computing judgment matrix weight vector}

The judgment matrix D regularization

$$
\bar{d}_{i j}=\frac{d_{i j}}{\sum_{k=1}^{n} d_{k j}}, j=1, \ldots, n
$$

Each column of the matrix which after normalization should according to line of adding together.

$$
\bar{Q}=\sum_{j=1}^{n} \bar{d}_{i j}, i=1, \ldots, n
$$

Vector $\bar{Q}=\left(\overline{q_{1}}, \overline{q_{2}}, \ldots, \overline{q_{n}}\right)$ regularization.

$$
q_{i}=\frac{\overline{q_{i}}}{\sum_{j=1}^{n} \overline{q_{j}}}
$$

Afterwards, it carries on the consistency check.If satisfied, this characteristic vector will namely judgment matrix weight vector.

\section{3) Consistency check}

Due to the construct judgment matrix sometimes cannot achieve the same, namely cannot make type (11) set up. So it needs to be consistency check:

$$
d_{i j}=\frac{d_{i k}}{d_{k j}},(i, j, k=1, \ldots, n)
$$

When checking the consistency of the judgment matrix, we first calculate the consistency index CI.

$$
C I=\frac{\lambda_{\max }-n}{n-1}
$$

In the formula, $\mathrm{n}$ is the number of rows or columns for the judgment matrix, and the $\lambda_{\max }$ is the maximum characteristic value.

By looking for the consistency index RI, we can calculate the consistency ratio $\mathrm{CR}$ :

$$
C R=\frac{C I}{R I}
$$

When CR $<0.1$, this judgment matrix satisfied Consistency check request, the solved characteristic vector can be used as static weight.

\section{B. Determine the Comprehensive Weight of the State Quantity}

Using entropy weight method to determine the index weight, in essence, it is to determine the weight value according to the amount of information of each index. The evaluation index of decision-making information were given the greater weight; On the other hand, it were given the small[14].

In the evaluation system of $m$ evaluation objects and $n$ evaluation index, it can form $\mathrm{M}^{*} \mathrm{~N}$-order evaluation matrix $R^{\prime}=\left[r_{i k}^{\prime}\right]_{m \times n}$, Where $r^{\prime}{ }_{i k}$ represents the value of the first $k$ evaluation index in the $i$ state model. After standardized treatment, the evaluation matrix $R=\left[r_{i k}\right]_{m \times n}$. The entropy of the K evaluation index is defined as:

$$
\varepsilon_{k}=-K \sum_{i=1}^{m} f_{i k} \ln f_{i k} \quad k=1,2, \ldots, n
$$

In the formula, $K=1 / \ln m$, $f_{i k}=r_{i k} / \sum_{i=1}^{m} r_{i k}$.

The entropy is the dynamic weight, and the static weight is modified to get the comprehensive weight:

$$
\lambda_{k}=\frac{\left(q_{k} / \varepsilon_{k}\right)}{\sum_{i=1}^{m} q_{k} / \varepsilon_{k}}
$$

Among them, $\mathrm{m}$ is the number of state variable.

\section{To Determine the Improved Approaching Degree}

The approaching degree accuracy determines the transformer health evaluation accuracy. The weight of each index is different, the role of its in the evaluation process is also different. The comprehensive weight of each state is determined by the static weight and the dynamic weight. Static weights reflect the relative importance of each state in the evaluation process. It consists of historical state and expert 
experiences. In this paper, dynamic weight uses entropy weight method to measure the change trend of the state. It reflects the influence of the change of the experimental data on the weight. Thus, the improved approaching degree is:

$$
R\left(\omega_{0}, \omega_{i}\right)=\lambda_{k} R\left(\omega_{0}(k), \omega_{i}(k)\right)
$$

It is more accurate than the traditional grey target theory to reflect the influence of each state variable of the approaching degree.

\section{CONCLUSION}

In this paper, considering various assessment index of $750 \mathrm{kV}$ transformer, we make the weighted grey target theory introduced into transformer insulation condition assessment, and use the Analytic Hierarchy Process (AHP) to determine the initial weights of each state variable, to distinguish the different contribution of the evaluation result. And the entropy weight is used to calculate the index of the degree of change to give the index weight, to modify the initial weight. So that more objective comprehensive evaluation results are obtained. According to the bull's-eye degree classification, we can effectively assess the health status of $750 \mathrm{kV}$ transformer, Provide appropriate treatment in time, in order to protect the normal operation of the system. This method, which is in the absence of standard fault model, can reflect the health status of the $750 \mathrm{kV}$ transformer better than the traditional grey target theory.

\section{REFERENCES}

[1] Zhang Deng. Research on condition assessment method for power transformer[D]. Wuhan: huazhong university of science and technology, 2013.

[2] Wu Guangning, Cui Yunguang, Wang Xiaojian. Evaluation of water content in insulation paper if different aging degrees using acid value in oil[j]. high-voltage technology, 2015,41 (1): 115-122.

[3] Ji Hongxia. Condition evaluationof power transformer based onimproved grey-target[D]. North China electric power university (baoding) of north China electric power university, 2010.

[4] Lakehal,A.;Ghemari,Z.;saad,S.Transformer fault diagnosis using dissolved gas analysis technology and Bayesian networks[J].Systems and Control, 2015:April 28-30.

[5] Mehta, A.K.; Sharma, R.N.; Chauhan, S.; Saho, S.Transformer diagnostics under dissolved gas analysis using Support Vector Machine[J]. Power, Energy and Control,2013:Feb 6-8.

[6] M.Farrokhi and M.Rafiee. Evaluation of Used and Repaired Power Transformers Using Neural Networks[C],Transmission and Distribution Conference and Exposition, 2001, 1: 143-146.

[7] Li xiaofeng. $750 \mathrm{kV}$ substation transformer on-line monitoring and fault diagnosis system design and application[D]. North China electric power university, 2014.

[8] Yang Lijun Study on Aging Characteristics of Oil-paper in Transformer and Its Lifetime Estimation Method[D]. Chongqing university, 2009.

[9] Lin Zhaohui Forecast of Transformer Life Time With Furfural Content[J]. Journal of transformer, 2009, 46 (7) : 35-38.

[10] Gong Senlian Study of life prediction model and reliability evaluation method for paper-oil insulation in power transformer [D]. Chongqing university, 2012.

[11] Cui Weinan The research on pavement preventive maintenance treatment based on weight coefficient grey target theory[D]. Hebei University of Technology, 2010.
[12] Li Jianpo Study on Power Transformer Fault Diagnosis Technology Based on Dissolved Gases Analysis[D]. Jilin university, 2008.

[13] Cao Jianjun The integrated evaluation of statement of transformer oilpaper insulation based on fuzzy analytic hierarchy process[D]. Southwest Jiaotong University, 2014.

[14] Wang Qi, Chang An, Song Yunhai, etc. A variable weights transmission equipment condition evaluation method based on combined weight theory[J]. Journal of China power, 2015, 48 (5) : 21-26. 